\title{
Two cases of lymphangitic carcinomatosis as the primary symptom of colorectal carcinoma that achieved complete remission using combination anti-EGFR antibody therapy
}

This article was published in the following Dove Medical Press journal:

OncoTargets and Therapy

Hirokazu Toshima

Toshikazu Ikusue

Atsushi Hisamatsu

Kouji Kobayashi

Ken Shimada

Medical Oncology, Showa University Koto Toyosu Hospital, Tokyo, Japan
Correspondence: Hirokazu Toshima Medical Oncology, Showa University Koto Toyosu Hospital, 5-I-38 Toyosu,

Koto-ku, Tokyo 135-8577, Japan

Tel +8I 362046342

Fax +8I 362046396

Email showa_northern@yahoo.co.jp

\begin{abstract}
Clinicians often encounter cases of pulmonary lymphangitic carcinomatosis when treating patients with cancer. When such a condition develops before the diagnosis of cancer, its diagnosis is often challenging. Herein, we report about two patients with colorectal carcinoma diagnosed after the identification of lymphangitic carcinomatosis, which achieved complete remission with combination anti-epidermal growth factor receptor (anti-EGFR) antibody therapy. In case 1, a 74-year-old woman presented with cough and dyspnea that had persisted for 1 month. She had unresectable advanced carcinoma of the sigmoid colon with lymphangitic carcinomatosis. Her respiratory status gradually deteriorated due to the disease. Thus, FOLFIRI plus cetuximab therapy was initiated. Her dyspnea rapidly resolved with the treatment, and complete remission of lymphangitic carcinomatosis was achieved. In case 2, a 46-year-old man presented with fever and dyspnea that had persisted for 1 month. He had unresectable advanced carcinoma of the transverse colon with lymphangitic carcinomatosis. FOLFOXIRI therapy was then initiated. However, his respiratory status did not improve. Therefore, his treatment was immediately switched to FOLFIRI plus panitumumab. His dyspnea rapidly resolved with the treatment, and complete remission of lymphangitic carcinomatosis was achieved. In oncologic emergencies, such as lymphangitic carcinomatosis, requiring an early response to treatment, the administration of anti-EGFR antibodies may be a highly effective treatment option.
\end{abstract}

Keywords: anti-epidermal growth factor receptor antibody, early tumor shrinkage, depth of response, chemotherapy, Pan-Asian adapted ESMO consensus guidelines, tumor location

\section{Introduction}

Clinicians often encounter cases of pulmonary lymphangitic carcinomatosis when treating patients with cancer. When the condition develops during cancer treatment or near the end of life, it can be immediately diagnosed based on its clinical course. However, when lymphangitic carcinomatosis develops before the diagnosis of cancer, its diagnosis is often challenging. The differential diagnosis includes pulmonary infection, pulmonary edema, interstitial pneumonia, sarcoidosis, pulmonary alveolar proteinosis, and so on. ${ }^{1}$ Numerous patients with lymphangitic carcinomatosis develop respiratory failure at the time of diagnosis, and emergency treatment is required in such cases. The prognosis for lymphangitic carcinomatosis is extremely poor, with a mortality rate of approximately $50 \%$ within 3 months of diagnosis based on one study. ${ }^{2}$ Therefore, immediate diagnosis and treatment are essential. Herein, we report two patients with colorectal carcinoma diagnosed after the identification (c)
hereby accept the Terms. Non-commercial uses of the work are permitted without any further permission from Dove Medical Press Limited, provided the work is properly attributed. For permission for commercial use of this work, please see paragraphs 4.2 and 5 of our Terms (https://www.dovepress.com/terms.php). 
of lymphangitic carcinomatosis, which achieved complete remission with combination anti-epidermal growth factor receptor (anti-EGFR) antibody therapy.

\section{Case reports}

A written informed consent was obtained from the patients for the publication of this case series along with their data. The institutional review board of Showa University Koto Toyosu Hospital does not require an institutional review for case reports.

\section{Case I}

Patient 1, a 74-year-old woman, presented with cough and dyspnea that had persisted for 1 month. The symptoms began mildly and then worsened. Rales were not audible on auscultation. Her abdomen was mildly distended, but not painful. Blood test results showed a mildly elevated white blood cell (WBC) count of 9,780 (normal range: 3,500-9,700)/ $\mathrm{LL}$ and C-reactive protein (CRP) level of 1.52 (normal range: $0-0.3) \mathrm{mg} / \mathrm{dL}$. The carcinoembryonic antigen (CEA) level was also elevated at 49.3 (normal range: $0-5.0) \mathrm{ng} / \mathrm{mL}$. Computed tomography (CT) scan revealed scattered nodules in both lungs, with thickening of the bronchovascular bundles and peripheral interlobular septa (Figure 1A). Marked thickening of the wall of the sigmoid colon was observed, indicating a primary tumor, and this was surrounded by a high-density area and air. Multiple nodules were also present in the lymph nodes and liver. Colonoscopy (CS) revealed a circumferential type 2 tumor in the sigmoid colon. Biopsy results revealed poorly differentiated adenocarcinoma, and results of the genetic screening showed wild-type RAS. Therefore, the patient was diagnosed with unresectable advanced carcinoma of the sigmoid colon with lymphangitic carcinomatosis.

Because microperforation of the sub-ileus was observed, decompression with a transanal ileus tube was started on the day of admission, and a transverse colostomy was performed without resecting the advanced carcinoma of the sigmoid colon on post-admission day 12. During this time, the patient's respiratory status gradually deteriorated, with an arterial oxygen partial pressure to fractional inspired oxygen $(\mathrm{PaO} 2 / \mathrm{FiO} 2)$ ratio that decreased to 273 . The patient was diagnosed with respiratory failure due to lymphangitic carcinomatosis, and treatment with dexamethasone $2 \mathrm{mg}$ /day (which is equivalent to prednisolone $13 \mathrm{mg}$ ) was started on day 15; however, the patient's dyspnea did not improve. Treatment with morphine slow-release tablet $20 \mathrm{mg} /$ day was also started on day 18, and the dose was temporarily increased to methylprednisolone $160 \mathrm{mg} /$ day, which is equivalent to prednisolone $200 \mathrm{mg}$. At this point, the patient's Eastern Cooperative Oncology Group Performance Status (ECOG PS) score was 3 , and the $\mathrm{PaO} 2 / \mathrm{FiO} 2$ ratio was 250 . After the risk had been fully explained to the patient and her family on day 24, FOLFIRI plus cetuximab therapy was initiated (irinotecan $150 \mathrm{mg} / \mathrm{m}^{2}$, bolus of $5-\mathrm{FU} 400 \mathrm{mg} / \mathrm{m}^{2}$, infusion with 5 -FU $2,400 \mathrm{mg} / \mathrm{m}^{2}$, leucovorin $200 \mathrm{mg} / \mathrm{m}^{2}$, and cetuximab $500 \mathrm{mg} / \mathrm{m}^{2}$ ). Dyspnea gradually improved immediately after chemotherapy on day $29(\mathrm{PaO} 2 / \mathrm{FiO} 2$ ratio $=319)$, and after the second course was started on day 38 , the patient's ECOG PS score had improved to 1. Dyspnea was resolved
Before treatment
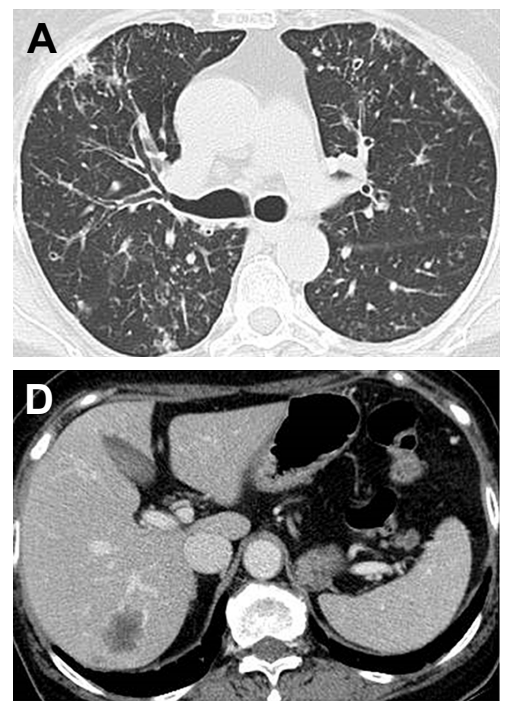

After the third course
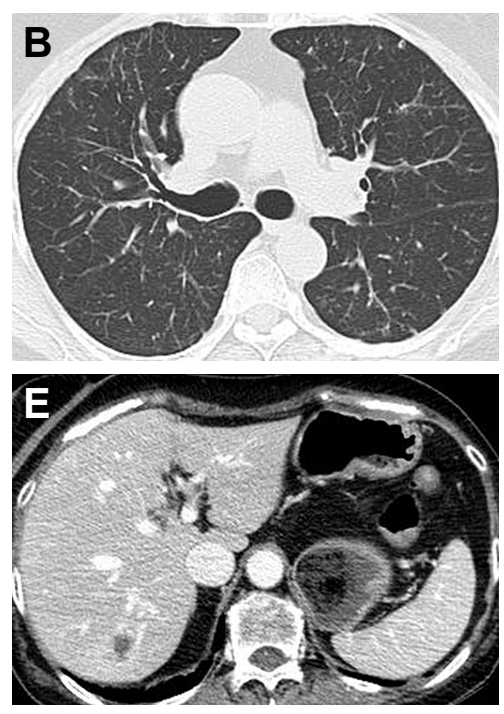

After the seventh course
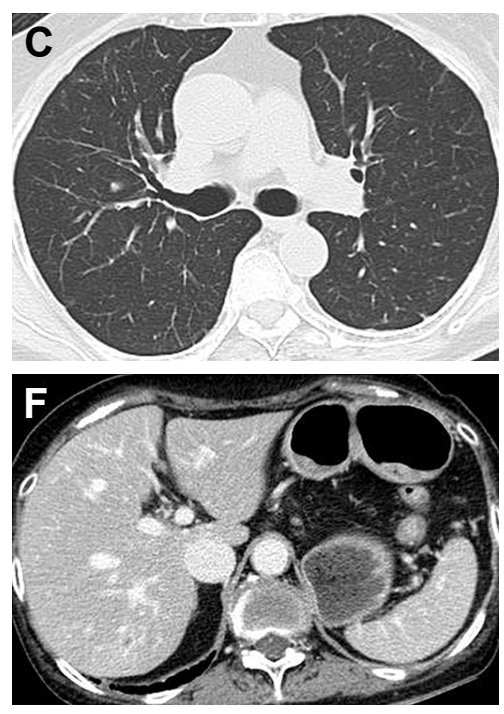

Figure I Computed tomography scans obtained (A and D) before and (B and E, C and F) after combination anti-EGFR antibody therapy in Case I. 
with such treatment. Thereby, the patient was discharged on day 43. Response evaluation CT scan after the third course showed that thickening of the peripheral interlobular septa in both lungs had improved. That is, only mild thickening was observed (Figure 1B); after the seventh course, thickening had disappeared completely (Figure 1C). Tumor shrinkage of the target lesions was also observed (Figure 1D-F), and no new lesion appeared. Along with the clinical complete remission of lymphangitic carcinomatosis, which is one of the non-target lesions, a partial response was observed after the initiation of combination anti-EGFR antibody therapy according to the Response Evaluation Criteria in Solid Tumors (RECIST) 1.1 criteria. Twenty months after the start of treatment, recurrence of lymphangitic carcinomatosis was not observed.

\section{Case 2}

Patient 2, a 46-year-old man, presented with fever and dyspnea that had persisted for 1 month. His dyspnea began mildly and then worsened. Coarse crackles were audible in both lung fields on auscultation. His abdomen did not appear distended, and it was not painful. Blood test results showed that his WBC count was $8,070 / \mu \mathrm{L}$, which is within the normal range. However, his CRP level was significantly elevated at $10.80 \mathrm{mg} / \mathrm{dL}$. The CEA and CA19-9 levels were also significantly elevated at $49.3 \mathrm{ng} / \mathrm{mL}$ and 318,190.1 (normal range: 0-37.0) $\mathrm{U} / \mathrm{mL}$, respectively. CT scan revealed scattered nodules and ground-glass shadow in both lungs, with thickening of the bronchovascular bundles and the peripheral interlobular septa (Figure 2A). Marked thickening of the intestinal wall near the hepatic flexure of the transverse colon that was indicative of a primary tumor was observed, and multiple nodules were also noted in the lymph nodes and liver. Moreover, CS revealed a circumferential type 2 tumor at the hepatic flexure of the transverse colon. Biopsy results revealed a poorly differentiated adenocarcinoma. The diagnosis was unresectable advanced carcinoma of the transverse colon with lymphangitic carcinomatosis.

Dyspnea was already severe at presentation, and the $\mathrm{PaO} 2 / \mathrm{FiO} 2$ ratio had decreased to 244 . The patient was diagnosed with respiratory failure and was then admitted. Oxygen therapy was initiated. His respiratory status continued to deteriorate after admission, and dexamethasone $2 \mathrm{mg} /$ day, which is equivalent to prednisolone $13 \mathrm{mg}$, was started on day 5. The patient was experiencing progressive respiratory failure due to lymphangitic carcinomatosis, and he had an ECOG PS score of 3. At this point, results of the RAS gene analysis were unknown. On day 9, after the risk had been fully explained to the patient and his family, FOLFOXIRI therapy was initiated (irinotecan $165 \mathrm{mg} / \mathrm{m}^{2}$, oxaliplatin $85 \mathrm{mg} / \mathrm{m}^{2}$, infusion with 5 -FU 3,200 mg/m², and leucovorin $200 \mathrm{mg} / \mathrm{m}^{2}$ ). However, his respiratory status did not improve after receiving FOLFOXIRI therapy, and the $\mathrm{PaO} 2 / \mathrm{FiO} 2$ ratio was 236. As the RAS genotype was found to be wild type, on day 17 panitumumab $6 \mathrm{mg} / \mathrm{kg}$ was added to his treatment. On day 23 , his treatment was switched to FOLFIRI plus panitumumab for the second course of therapy, and his dyspnea gradually improved $(\mathrm{PaO} 2 / \mathrm{FiO} 2$ ratio $=338)$; on day 33, his ECOG PS score improved to 1. This treatment sufficiently improved the patient's subjective symptoms, and

\section{Before treatment}
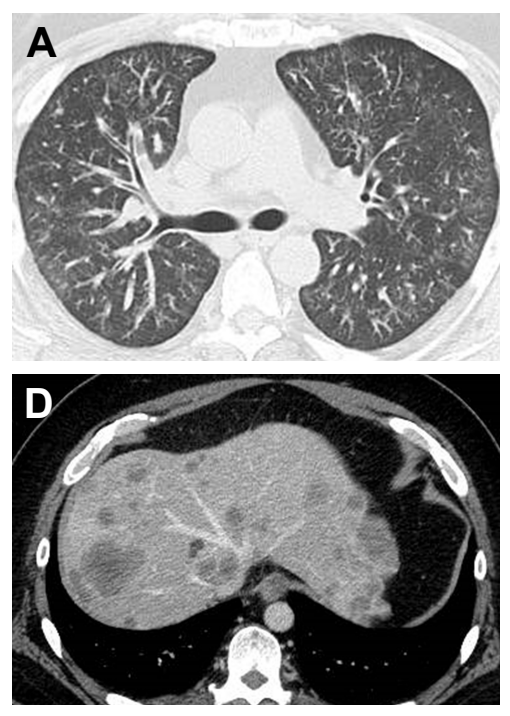

After the second course
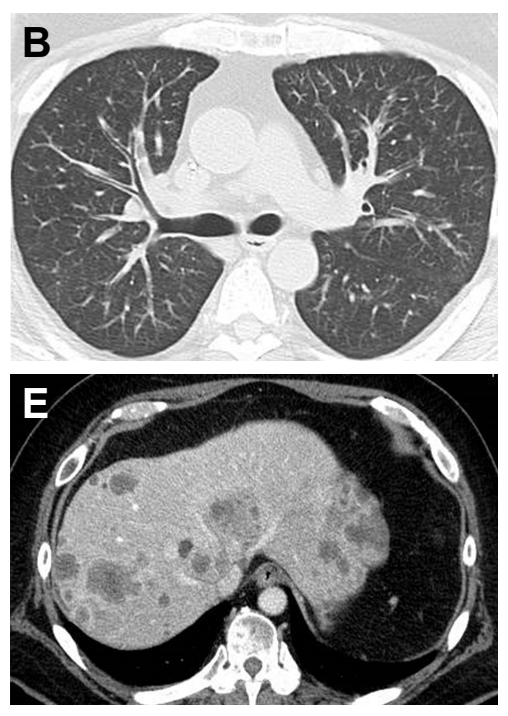

After the fourth course
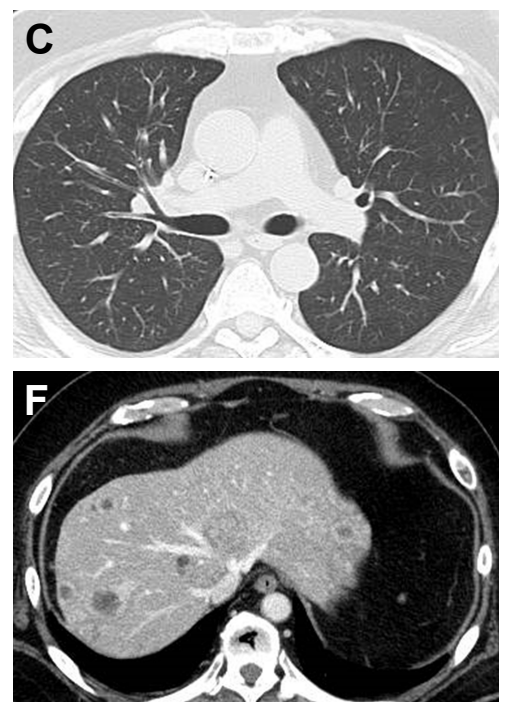

Figure 2 Computed tomography scans obtained ( $\mathbf{A}$ and $\mathbf{D})$ before and (B and $\mathbf{E}, \mathbf{C}$ and $\mathbf{F})$ after combination anti-EGFR antibody therapy in Case 2. 
he was discharged on day 41. Response evaluation CT scan after the second course showed that the ground-glass shadows in both lungs had disappeared, and significant improvement in the thickening of the bronchovascular bundles and peripheral interlobular septa was observed (Figure 2B). After the fourth course, clinical complete remission of lymphangitic carcinomatosis had been achieved (Figure 2C). Tumor shrinkage of the target lesions was also noted (Figure 2D-F), and no new lesion appeared. Along with the clinical complete remission of lymphangitic carcinomatosis, which is one of the non-target lesions, a partial response was observed after the initiation of combination anti-EGFR antibody therapy according to the RECIST 1.1 criteria. Eight months after the start of treatment, recurrence of lymphangitic carcinomatosis was not observed.

\section{Discussion}

The Pan-Asian Adapted European Society for Medical Oncology (ESMO) Consensus Guidelines state that clinical evaluation must first be conducted when treating patients with advanced colorectal cancer. ${ }^{3}$ If a patient is eligible for chemotherapy, then treatment strategy is chosen, with the therapeutic goal of either cytoreduction or disease control. Respiratory failure due to lymphangitic carcinomatosis makes it extremely challenging to determine whether cancer drug therapy is indicated based on the patient's systemic condition. However, if a patient is eligible for chemotherapy, cytoreduction is set as the goal due to its urgency.

Both of our patients had ECOG PS scores of 3 at the time of definitive diagnosis. Drug therapy for solid tumors is usually only provided to patients with an ECOG PS score of 0-2, and its therapeutic indications for patients with an ECOG PS score of 3 are limited. In patients with a poor general condition (an ECOG PS score of 3 or 4), the risk of treatment-related death is high. ${ }^{4,5}$ However, in both of our patients, a poor PS was due to the presence of lymphangitic carcinomatosis; we believed that if lymphangitic carcinomatosis could be controlled, their PS was more likely to improve. In general, first-line, combination molecular targeted drug therapy is frequently used to treat patients with colorectal carcinoma due to a high response rate. In cases similar to our patients, the high sensitivity and immediate response to cancer drug therapy for primary carcinoma is important. If such therapy is available, then eligible patients can receive the therapy.

A pooled analysis of the prognostic and predictive value by the location of the primary colorectal carcinoma lesion (left-sided versus right-sided) for the treatment of patients with wild-type RAS was conducted. ${ }^{6}$ In this report, the benefit of combination anti-EGFR antibody therapy was limited in the treatment of patients with right-sided carcinoma. According to the ESMO Consensus Guidelines based on this analysis, the following treatment algorithm is used, depending on the location of the primary colorectal carcinoma lesion. For patients with wild-type RAS in whom the goal is cytoreduction, the recommended treatment for left-sided carcinoma is two cytotoxic chemotherapy agents plus an anti-EGFR antibody; meanwhile, the recommendation for right-sided carcinoma is either three cytotoxic chemotherapy agents or two of such agents plus bevacizumab. Three cytotoxic chemotherapy agents plus bevacizumab might have been an effective treatment option for patient 2 who had right-sided carcinoma.

The results of the FIRE-3 and the PEAK trials are also important, which indicate that anti-EGFR antibodies may result in early tumor shrinkage and depth of response. ${ }^{7,8}$ In patients with respiratory failure due to lymphangitic carcinomatosis, an early response to treatment is essential, and we considered that anti-EGFR antibodies would provide rapid, deep tumor shrinkage. The efficacy of combination therapy, including treatment with anti-EGFR antibody, among Japanese patients in the JACCRO CC-05/06 trials was investigated. ${ }^{9,10}$ An additional study based on those trials has found that some patients with right-sided carcinoma may also benefit from the addition of an anti-EGFR antibody. ${ }^{11}$ Based on such findings, both patients were treated with an anti-EGFR antibody. As a result, dyspnea immediately improved even in patient 2 , who had right-sided carcinoma, and complete remission of lymphangitic carcinomatosis was achieved.

\section{Conclusion}

Respiratory failure due to lymphangitic carcinomatosis is a so-called oncologic emergency that requires urgent treatment. In such cases, the treatment strategy must be considered not only with respect to simple data on the location of the primary colorectal carcinoma lesion, which may provide a surrogate for its oncobiological characteristics, but also in light of the pharmaceutical characteristics. We used combination antiEGFR antibody therapy to treat two patients with colorectal carcinoma who had respiratory failure due to lymphangitic carcinomatosis at the time of diagnosis, but achieved complete remission of the lymphangitic carcinomatosis. In oncologic emergencies requiring an early response to treatment, the rapid and deep tumor shrinkage provided by anti-EGFR antibodies may be a highly effective treatment option. 


\section{Acknowledgment}

We would like to thank Editage (www.editage.jp) for English language editing.

\section{Disclosure}

Ken Shimada has received research grants from Chugai Pharmaceutical, Taiho Pharmaceutical and Medicon Incorporated. The other authors report no conflicts of interest in this work.

\section{References}

1. De Wever W, Meersschaert J, Coolen J, Verbeken E, Verschakelen JA. The crazy-paving pattern: a radiological-pathological correlation. Insights Imaging. 2011;2:117-132.

2. Bruce DM, Heys SD, Eremin O. Lymphangitis carcinomatosa: a literature review. J R Coll Surg Edinb. 1996;41:7-13.

3. Yoshino T, Arnold D, Taniguchi H, et al. Pan-Asian adapted ESMO consensus guidelines for the management of patients with metastatic colorectal cancer: a JSMO-ESMO initiative endorsed by CSCO, KACO, MOS, SSO and TOS. Ann Oncol. 2018;29:44-70.

4. Ohe Y, Yamamoto S, Suzuki K, et al. Risk factors of treatment-related death in chemotherapy and thoracic radiotherapy for lung cancer. Eur J Cancer. 2001;37:54-63.

5. Wheatley-Price P, Ali M, Balchin K, Spencer J, Fitzgibbon E, Cripps C. The role of palliative chemotherapy in hospitalized patients. Curr Oncol. 2014;21:187-192.
6. Arnold D, Lueza B, Douillard JY, et al. Prognostic and predictive value of primary tumour side in patients with RAS wild-type metastatic colorectal cancer treated with chemotherapy and EGFR directed antibodies in six randomized trials. Ann Oncol. 2017;28:1713-1729.

7. Stintzing S, Modest DP, Rossius L, et al. FOLFIRI plus cetuximab versus FOLFIRI plus bevacizumab for metastatic colorectal cancer (FIRE-3): a post-hoc analysis of tumour dynamics in the final RAS wild-type subgroup of this randomised open-label phase 3 trial. Lancet Oncol. 2016;17:1426-1434.

8. Rivera F, Karthaus M, Hecht JR, et al. Final analysis of the randomised PEAK trial: overall survival and tumour responses during first-line treatment with mFOLFOX6 plus either panitumumab or bevacizumab in patients with metastatic colorectal carcinoma. Int J Colorectal Dis. 2017;32:1179-1190.

9. Tsuji A, Sunakawa Y, Ichikawa W, et al. Early tumor shrinkage and depth of response as predictors of favorable treatment outcomes in patients with metastatic colorectal cancer treated with FOLFOX plus cetuximab (JACCRO CC-05). Target Oncol. 2016;11:799-806.

10. Sunakawa $Y$, Ichikawa W, Tsuji A, et al. Prognostic impact of primary tumor location on clinical outcomes of metastatic colorectal cancer treated with cetuximab plus oxaliplatin-based chemotherapy: a subgroup analysis of the JACCRO CC-05/06 trials. Clin Colorectal Cancer. 2017; 16:e171-e180.

11. Sunakawa Y, Tsuji A, Fujii M, Ichikawa W. No benefit from the addition of anti-EGFR antibody in all right-sided metastatic colorectal cancer? Ann Oncol. 2017;28:2030-2031.
OncoTargets and Therapy

\section{Publish your work in this journal}

OncoTargets and Therapy is an international, peer-reviewed, open access journal focusing on the pathological basis of all cancers, potential targets for therapy and treatment protocols employed to improve the management of cancer patients. The journal also focuses on the impact of management programs and new therapeutic agents and protocols on

\section{Dovepress}

patient perspectives such as quality of life, adherence and satisfaction. The manuscript management system is completely online and includes a very quick and fair peer-review system, which is all easy to use. Visit http://www.dovepress.com/testimonials.php to read real quotes from published authors. 